\title{
X-linked ichthyosis and Crigler-Najjar syndrome I: Coexistence in a male patient with two copy number variable regions of $2 q 37.1$ and Xp22.3
}

\author{
JINLI BAI*, YUJIN QU* , YANYAN CAO, YAN LI, WENHUI ZHANG, YUWEI JIN, HONG WANG and FANG SONG \\ Department of Medical Genetics, Capital Institute of Pediatrics, Beijing 100020, P.R. China
}

Received January 26, 2015; Accepted November 18, 2015

DOI: $10.3892 / \mathrm{mmr} .2015 .4674$

\begin{abstract}
X-linked ichthyosis (XLI) is an X-linked recessive skin disorder generally restricted to males, which arises from mutations in the steroid sulfatase (STS) gene located on $\mathrm{Xp22.3.} \mathrm{Crigler-Najjar} \mathrm{syndrome} \mathrm{(CN-I)} \mathrm{is} \mathrm{a} \mathrm{rare} \mathrm{autosomal}$ recessive disease caused by the homozygous or compound heterozygous mutations in the UPD-glucuronosyltransferase 1 family, polypeptide A1 (UGT1A1) gene on chromosome 2q37. A male patient was referred to the Department of Medical Genetics with of severe icterus and ichthyosis. The patient and his family members underwent genetic tests related to XLI and CN-I. Quantitative polymerase chain reaction on genomic DNA was performed to determine the gene copy number, while single nucleotide polymorphism array analysis was conducted to identify deletion mutations. Family pedigree analysis showed that the patient and his two cousins were all affected by ichthyosis, which was in accordance with the inheritance pattern of an X-linked recessive disease. In addition, the patient's serum bilirubin concentration (>340 mmol/l) was markedly greater than the normal level. The patient presented with kernicterus and phenobarbital treatment was ineffective. The clinical diagnosis of XLI was confirmed molecularly by laboratory evidence of a maternal 1.61 M deletion (including the STS gene) on ChrXp22.31. Coincidentally, the male patient was also confirmed to carry a rare maternal inherited microdeletion $(374 \mathrm{~Kb}$ ) comprising the entire UGT1A1 gene combined with a paternal UGT1A1 mutation (c.1253delT), a causative
\end{abstract}

Correspondence to: Professor Fang Song, Department of Medical Genetics, Capital Institute of Pediatrics, 2 Yabao Road, Chaoyang, Beijing 100020, P.R. China

E-mail: songf_558@263.net

${ }^{*}$ Contributed equally

Abbreviations: CN-I, Crigler-Najjar syndrome; UGTIAl gene, UPD-glucuronosyltransferase 1 family, polypeptide A1 gene; XLI, X-linked ichthyosis; STS, steroid sulfatase; CNVs, copy number variations; SNP, single nucleotide polymorphism

Key words: X-linked ichthyosis, Crigler-Najjar syndrome, 2q37.1, Xp22.31, copy number variation event of CN-I. To the best of our knowledge, this study reported for the first time the comorbidity of XLI and CN-I in a male patient. The results suggested that co-occurrence of these two recessive diseases in a patient may be incidental.

\section{Introduction}

X-linked ichthyosis (XLI, OMIM\#308100) is an X-linked recessive skin disorder that is caused by steroid sulfatase (STS) deficiency (1). The incidence rate of XLI is 1 in 6,000 males studied in various populations (1). It is characterized by desquamation of large brown scales on the limb and trunk (2). Extracutaneous manifestations, particularly corneal opacities and cryptorchidism, are frequently observed (3). The disease is caused by mutations in the STS gene that is located on the short arm of the X-chromosome (Xp22.3) (4). Approximately $90 \%$ of patients with XLI have large deletions involving the entire STS gene and flanking regions $(5,6)$.

Crigler-Najjar syndrome type I (CN-I, MIM\#218800) is a rare autosomal recessive disease caused by the genetic alterations in the UPD-glucuronosyltransferase 1 family, polypeptide A1 (UGT1A1) gene (7). It is characterized by high levels of serum total bilirubin (342-684 $\mu \mathrm{mol} / \mathrm{l})$ caused by the complete absence of the UGT1A1 enzyme, with a frequency of less than one per million live births (8). The accumulation of unconjugated bilirubin in the serum may cause irreversible neurological damage (kernicterus). The brain damage can be prevented by intensive phototherapy that is effective at least until adolescence (9). However, CN-I is a lethal disease when not treated adequately and phenobarbital treatment is not effective for the treatment of symptoms. Liver transplantation is the only definitive treatment for CN-I. To date, the majority of UGT1A1 mutations are single nucleotide substitutions, including missense mutations and nonsense mutations. Large deletions or insertions of UGT1A1 gene are less reported.

Human genetic diseases are predominantly associated with DNA variations, ranging from single nucleotide changes to large DNA fragment variations, such as copy number variations (CNVs). XLI and CN-I are two genetic diseases with Mendelian inheritance patterns, however, they have distinct pathogenic mechanisms and clinical manifestations. XLI predominantly arises from CNVs involving the STS gene on the Xp22.3 region. By contrast, CN-I is usually the result of point mutations in the UGTIAl gene, and CNVs involving this 
gene have rarely been reported. Moreover, a coexistence of the two genetic alterations has not been reported.

The present study presents a case of a Chinese male patient affected by recessive XLI and CN-I, which were confirmed by DNA quantitative polymerase chain reaction (PCR) and single nucleotide polymorphism (SNP) array analysis. To the best of our knowledge, this is the first report this co-morbidity.

\section{Materials and methods}

DNA samples. Genomic DNA samples were extracted from the peripheral blood leukocytes of the patient and his parents, using the phenol-chloroform method (10). Informed consent was obtained from the patient's parents. This project was approved by the ethics committee of the Capital Institute of Pediatrics (Beijing, China).

Quantitative PCR for STS gene-copy number analysis. As $90 \%$ of the patients with XLI have large deletions involving the entire STS gene, the copy numbers of this gene were directly detected by quantitative PCR on genomic DNA. Primers were designed using the Primer Express 3.0 software (Applied Biosystems, Thermo Fisher Scientific, Inc., Waltham, MA, USA). The STS primers used were as follows: STS-E1F, 5'-GTT GCCCCTTCTGAAGAATCC-3'; STS-E1R, 5'-GAGGCG GAGACACTCTTTGC-3'; STS-E10F, 5'-TGGAGTGCAGTG GTGCAATC-3'; STS-E10R, 5'-GTGACTTGGGAGGCTGAA GTG-3'. The quantitative PCR was conducted using the ABI Prism 7500 sequence detection system (Applied Biosystems, Thermo Fisher Scientific Inc.) as described by Wilke et al (11). Data were analyzed using the ABI 7500 Prism sequence detection software (Applied Biosystems, Thermo Fisher Scientific, Inc.). Glyceraldehyde 3-phosphate dehydrogenase (GAPDH) was used as an internal control gene for gene copy number analysis. The $2^{-\Delta \Delta \mathrm{Cq}}$ method for relative quantification was used to evaluate STS gene copy numbers of the patient and his parents in comparison with those of healthy controls. DNA from healthy controls served as a calibrator that had a $2^{-\Delta \Delta \mathrm{Cq}}$ of 1 . The $2^{-\Delta \Delta \mathrm{Cq}}$ value of the inspected sample is the ratio of the target gene copy number of inspected sample to the calibrator.

SNP array analysis. A genome-wide SNP array, Human CytoSNP-12 DNA Analysis BeadChip kit (Illumina, Inc., San Diego, CA, USA) was used to examine the deletion sizes of the two regions (2q37.1 and Xp22.31) containing STS and UGT1A1 genes. The genome-wide SNP array was composed of 300,000 probe markers. Briefly, $4 \mu 1$ DNA sample (200 ng) was mixed with $4 \mu \mathrm{l} \mathrm{NaOH}(0.1 \mathrm{~mol} / \mathrm{l})$ solution, followed by a 10-min incubation at room temperature for DNA denaturation. Next, $68 \mu 1$ MA2 solution and $75 \mu 1$ MSM solution (both from Illumina, Inc.) were added to the mixture to allow DNA renaturation. The reaction solution was put in the $37^{\circ} \mathrm{C}$ hybrid furnace for $20 \mathrm{~h}$. The resulting DNA fragments were resuspended in RA1 solution (Illumina, Inc.). and the DNA solution was loaded onto the chip. The chip was kept in an airtight hybrid box (Illumina, Inc.) to permit hybridization for $16 \mathrm{~h}$ at $48^{\circ} \mathrm{C}$. When the chip was washed to remove non-hybridized and nonspecific hybridized DNA fragments, single base extension and chip staining reactions were initiated. The iScan scanning system (Illumina, Inc.) was used to collect data, and the data were further analyzed by Karyostudio software v1.4 (Illumina, Inc.).

\section{Results}

Clinical presentation. The male patient is the first offspring of healthy and nonconsanguineous parents. He was born at term by normal delivery; however, his mother had the premonitory symptoms of miscarriage two months into the pregnancy. The patient presented with yellowing of the skin 6 days after birth. He was admitted to the Capital Institute of Pediatrics due to yellowing of the skin that did not reduce at 4.5 months old. His total serum bilirubin level was $520.6 \mu \mathrm{mol} / 1$ and serum unconjugated bilirubin level was $499.6 \mu \mathrm{mol} / \mathrm{l}$. The patient succumbed to kernicterus at 13 months of age, due to molecularly confirmed CN-I syndrome (12). However, upon reviewing the related medical records, other abnormal manifestations in the patient that could not be fully attributed to CN-I alone, were identified. Upon admission, the physical examination indicated that the patient was 7,000 $\mathrm{g}$ (20th) in weight and $66 \mathrm{~cm}$ in length (50-75th), with a head circumference of $41 \mathrm{~cm}$ (20th), which revealed that the patient had growth retardation. He could not raise his head at 4 months old of age, which suggested slight retardation of mental and motor development. A dispersed congestive rash were observed on his face and head, and rhombus-shaped brown pigmentation and furfuration were observed on his trunk and four limbs. A follow-up survey with the family revealed that the patient's two cousins also suffered from ichthyosis (Fig. 1); thus, XLI was suspected. Apart from ichthyosis, the patient had inspiratory stridor after birth and was diagnosed with congenital laryngeal cartilage softening disease. Abdominal computer tomography scanning indicated that the patient had left renal dysplasia and left renal artery stenosis. However, his renal function and routine urine test results were normal.

Gene copy number changes. For the STS gene, the patient's mother and father both carried only one copy number $(\mathrm{N}=1)$, although the male patient did not carry the gene $(\mathrm{N}=0)$. The results of real-time quantitative PCR are shown in Fig. 2, and the $2^{-\Delta \Delta \mathrm{Cq}}$ value of the control (male or female) was considered to be 1 .

The 'homozygous mutation' of c.1253delT in exon 4 of the UGT1Al gene was detected in the patient by DNA sequencing in our previous study (12). The same mutation was observed in his father, but not in his mother. It is possible that an allele lacking the entire UGTIAl gene may be transmitted to the patient from the mother who had a single UGT1A1 gene copy. Therefore, for the patient with one point mutation (c.1253delT) on one allele and with an entire deletion of the UGT1Al gene on the other allele, the possibility existed of falling into the 'trap' of 'homozygous mutation' (c.1253delT) in the patient by direct DNA sequencing. Thus, quantitative PCR was performed to detect the copy number of UGT1Al gene in the core members. The results demonstrated that his father carried two copies of the UGT1A1 gene, with c.1253delT detected on one of alleles. His mother only had one copy of the UGTIAl gene without base changes. The patient also carried a single copy of the UGTIAl gene that harbored the c.1253delT mutation. 
A

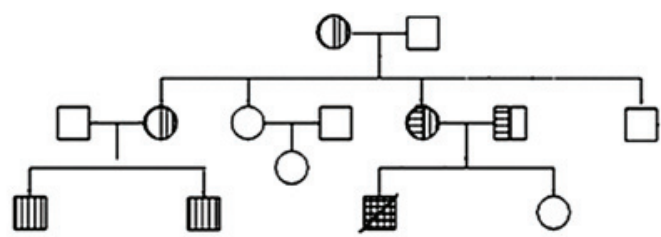

B

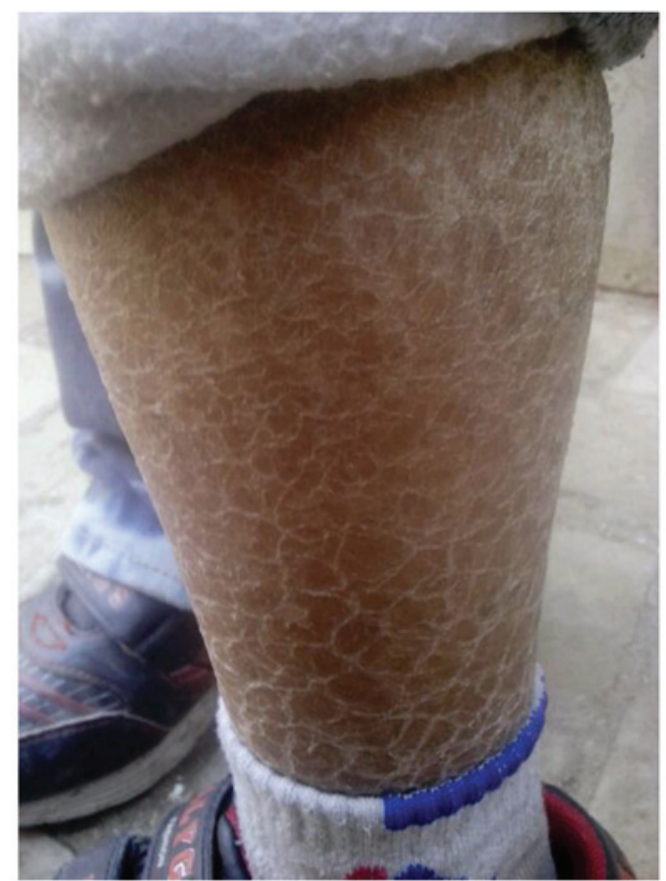

Figure 1. (A) Pedigree analysis of CN-I and XLI. White square, normal male; white circle, normal female; circle right filled with vertical lines, female carrier with XLI; square filled with vertical lines: male patient with XLI; circle left filled with horizontal lines and right filled with vertical lines: female carrier with CN-I and XLI; square left filled with horizontal lines: male carrier with CN-I; square filled with vertical lines and horizontal lines: the male proband with XLI; diagonal line, death. (B) Rhombus-shaped brown pigmentation on the lower limbs of the patient's cousin with XLI. CN-I, Crigler-Najjar syndrome; XL1, X-linked ichthyosis.

Deletion range detection. SNP array results are shown in Fig. 3. Two CNVs were confirmed for this patient: One maternal microdeletion was located on ChrXp22.31 with an estimated size of $1.61 \mathrm{M}$; the other maternal microdeletion was found on Chr2q37.1 (Table I), with an estimated size of 374-kb (Fig. 3A and B). Moreover, SNP array results showed that his mother had the same microdeletion of $374 \mathrm{~kb}$ on Chr2q37.1, but a larger deletion $(\sim 1.67 \mathrm{Mb})$ on ChrXp22.31, including STS and additional five genes (for example, mental retardation related gene VCX3A) (Fig. 3C and D). Thus, these data suggested that the two CNVs in the patient may be inherited from his mother. One CNV contained an XLI-causing gene, STS, and the other $\mathrm{CNV}$ comprised a causative gene for CN-I, UGTIAl. These genetic abnormalities were well matched to the main clinical phenotypes of the patient, i.e, ichthyosis and icterus (Table I).

\section{Discussion}

In this study, a unique patient with XLI and CN-I, two recessive inherited diseases, was described. The two diagnoses were confirmed by molecular genetic evidence, as the patient exhibited a maternal $1.61 \mathrm{Mb}$ deletion involving the STS
A

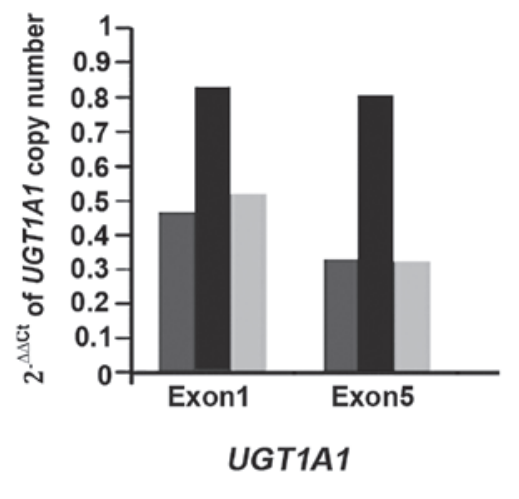

B

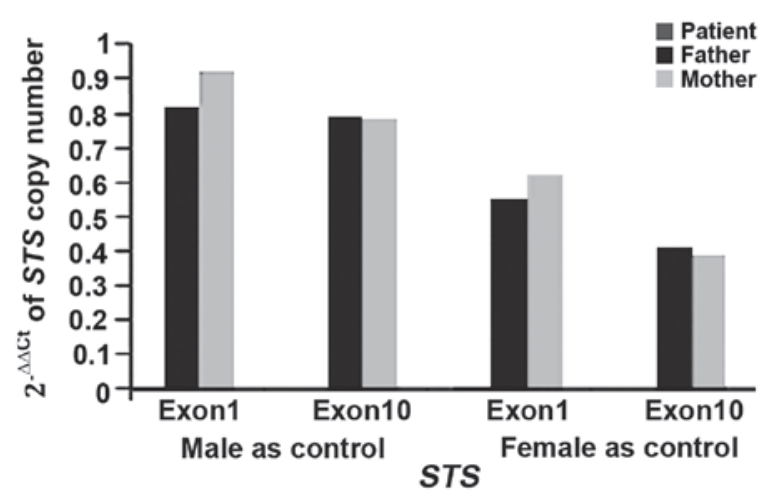

Figure 2. Quantitative polymerase chain reaction result of UGT1A1 and STS. (A) UGT1A1 gene in the patient and his parents; (B) STS gene in the patient and his parents. UGT1A1, UPD-glucuronosyltransferase 1 family, polypeptide A1; STS, steroid sulfatase.

gene, and a $374 \mathrm{~Kb}$ maternal deletion involving the UGT1Al gene combined with a paternal UGT1Al gene mutation (c.1253delT). Since these two recessive inherited diseases existed simultaneously in the same patient but independently with each other, it was speculated that the co-occurrence of two conditions was incidental. To the best of our knowledge, this is the first reported case with this comorbidity.

The patient's clinical symptoms of the skin were consistent with the diagnosis of ichthyosis. Pedigree analysis showed that there were two affected cousins in the extended family, which was consistent with the transmission characteristics of X-linked recessive diseases. Up to $90 \%$ of XLI patients have large gene fragment deletions involving the entire STS gene and its flanking regions, while the minorities of cases display partial deletions or point mutations in the gene $(5,6,13)$. In this study, quantitative PCR and SNP microarray analysis confirmed the deletion of the entire STS gene in this patient. A previous study demonstrated that this aberration was the result of the unequal crossing-over in the two regions between the highly conserved CRI-S232 repeat regions flanking the STS gene (14). The deletion size of XLI in this patient was $1.61 \mathrm{Mb}$, which was close to the common $1.5-\mathrm{Mb}$ deletion. Recent studies have shown that nervous system abnormalities, short stature, mental retardation, Kallmann syndrome or other clinical features may occur, when the deletion size on Xp22.3 is $>1.5 \mathrm{Mb}(15,16)$. Accordingly, the $V C X 3 A$ gene located $\sim 0.7 \mathrm{Mb}$ telomeric to the $S T S$ gene has been proposed as the candidate mental retardation gene on Xp22.3 (17,18). In the present study, the STS and VCX3A genes were deleted on 
Table I. Deletion result of single nucleotide polymorphism array analysis of the patient.

\begin{tabular}{lccclc}
\hline Region & From & To & Length (bp) & \multicolumn{1}{c}{ Gene } & Related \\
phenotype
\end{tabular}

UGT1A1, UPD-glucuronosyltransferase 1 family, polypeptide A1; STS, steroid sulfatase.

A

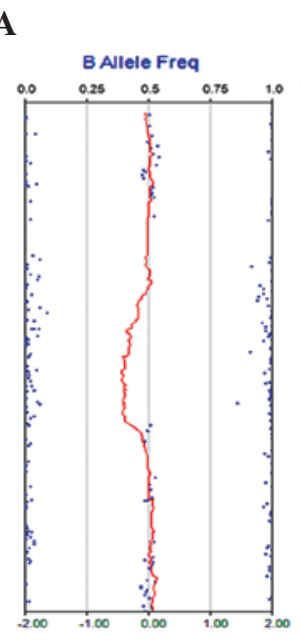

C

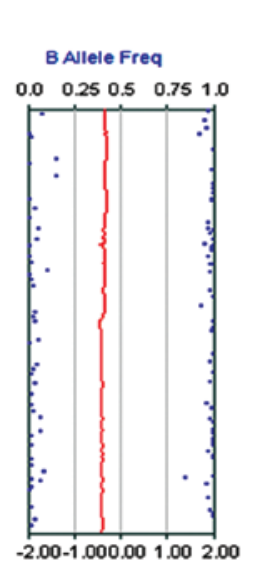

登?

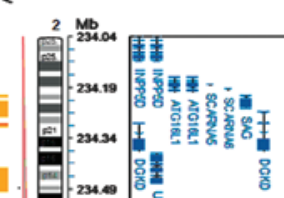

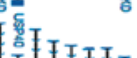

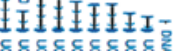

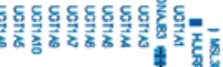

$\frac{1}{3}$

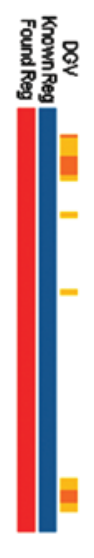

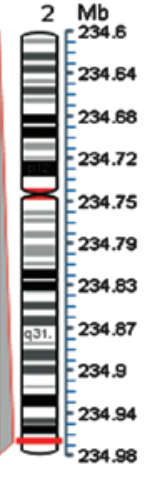

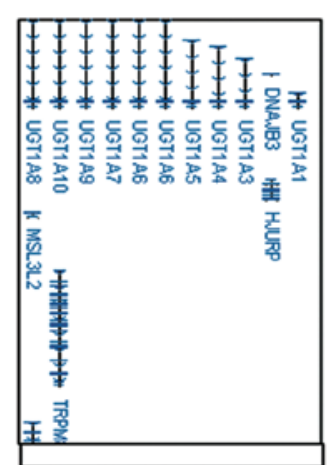

D

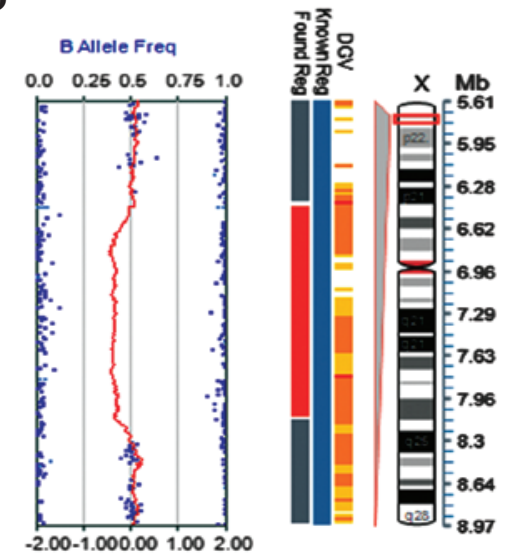

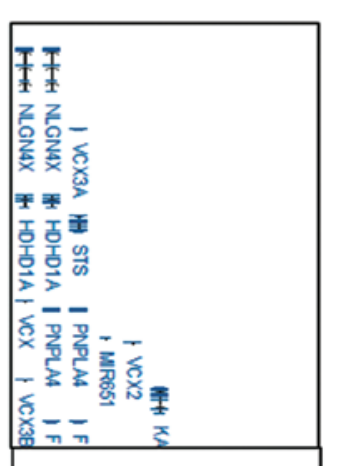

Figure 3. Single nucleotide polymorphism results of the patient and his mother. (A) Chromosome 2 of the patient; (B) chromosome X of the patient; (C) chromosome 2 of the patient's mother; (D) chromosome $\mathrm{X}$ of the patient's mother.

one of the $\mathrm{X}$ chromosomes of the patient's mother who had a normal phenotype. By contrast, the male patient presented with slight mental retardation, although he only lost the STS gene, but not the $V C X 3 A$ gene. Such a disconnection of genotype and phenotype was considered to be due to the fact that number and location of the probes and hybridization signals of this region may limit the capacity to determine the accurate deletion size by SNP analysis. Unfortunately, we failed to repeat the SNP analysis or conduct another experiment to confirm the results, due to the lack of availability of the patient DNA samples. In addition, although the deletion range of the patient did not include the Kallmann syndrome 1 protein, certain clinical manifestations of Kallmann syndrome were observed, such as left renal dysplasia. Conversely, he did not have the most common symptoms of XLI (corneal opacity or cryptorchidism). Moreover, the pathogenic mechanism for other complex manifestations, such as congenital laryngeal cartilage softening disease, require further elucidation.

To date, $>130$ UGT1A1 gene mutations have been identified, including 91 single nucleotide changes (77 missense mutations and 14 nonsense mutations), 21 deletions, 10 insertions and 8 mutations located in the promoter and introns (19). 
Complete loss of the UGT1A1 gene is rarely reported. The present study initially performed direct DNA sequencing to screen the mutations, based on the feature of the UGT1Al gene mutation spectrum. This result revealed that the patient had a homozygous mutation-c.1253 Del T of the UGT1AI gene, which could not be fully explained by mutation transmission analysis of his parents as the same mutation was only observed in his father and not in his mother. Thus, this phenomenon prompted us to further careful analysis of DNA sequencing data for UGT1A1, the causative gene of the monogenic disease. Since the parents of the patient were unconsanguineous, it was presumed that the patient's mother only carried one copy of normal UGTIAl gene as indicated by DNA sequence analysis. Subsequently, PCR and SNP microarray analysis confirmed that the mother only had a single copy of UGTIAl gene. It is extremely rare for CN-I to be caused by a point mutation and a complete deletion of the UGT1A1 gene. As the clinical phenotypes of this patient were more complex than what the known genotypes could explain, it was necessary to analyze the deletion range to understand the correlation between genotype and phenotype. Therefore, a Genome-wide SNP array analysis was conducted in this study.

CNV is a form of DNA structural variation ( $\geq 1 \mathrm{~kb})$, which is present at a variable copy number, when compared with a reference genome. It occurs by recombination-based and replication-based mechanisms (18). Although CNVs can represent benign polymorphic variants widely distributed in the human genome, there are numerous CNVs that can cause Mendelian or sporadic traits, or be associated with complex diseases (20). To assess the pathogenicity of CNVs, the ISCA Consortium has published the criteria in their consensus statement of 2010 (21). For example, if the CNVs contain morbid MIM genes, they are probably pathogenic. By contrast, when the CNVs are inherited from a healthy parent, they are probably benign (22). In this study, the proband has been confirmed to carry two CNVs: One was a $374 \mathrm{~Kb}$ microdeletion located on Chr2q37.1 and the other was $\sim 1.61 \mathrm{M}$ deletion located on ChrXp22.31. The two CNVs contained two disease-causing genes, UGT1A1 and STS, respectively. Family pedigree analysis showed that the two CNVs were inherited from the patient's mother. It was assumed that the $1.61 \mathrm{M}$ deletion may be transmitted from the proband's grandmother as both cousins exhibited the phenotype of ichthyosis. Furthermore, a $374 \mathrm{~kb}$ microdeletion that resulted in CN-I was passed from the proband's mother. However, it could not be determined whether this microdeletion was inherited from the grandparents or was de novo in his mother. Cases with the two recessive genetic diseases have not been reported. The CN-I of the patient in the present study resulted from a rare $374 \mathrm{~Kb}$ maternal microdeletion combined with a paternal c. 1253 delT mutation. Coincidentally, the proband was a male and also carried the maternal 1.61 M deletion located on ChrXp22.31, which caused him to lose the STS gene and consequentially develop XLI.

In conclusion, this study suggested that rare diseases may not simply be associated with the affected gene but may also be concurrent with genomic variations, such as CNVs. It also highlighted the importance of conducting detailed clinical examinations and unbiased genetic analyses, particularly for the patients presenting with clinical phenotypes that could not be fully explained by general genetic testing results. Further studies using genome-wide scanning techniques (e.g., SNP array) may aid in determining disease-causing genetic alterations to understand the phenotypes in the affected individuals. Gaining a full understanding of the phenotype may assist in enabling a more accurate diagnosis to be made, and also in providing an improved basis for rational treatment strategies.

\section{Acknowledgements}

The authors would like to thank the family members for participating. They would also like to acknowledge Dr Gao Ying (Department of Dermatology, Capital Institute of Pediatrics) for providing helpful comments.

\section{References}

1. Shapiro LJ, Weiss R, Webster D and France JT: X-linked ichthyosis due to steroid-sulphatase deficiency. Lancet 14: 70-72, 1978.

2. Hosomi N, Oiso N, Fukai K, Hanada K, Fujita H and Ishii M: Deletion of distal promoter of VCXA in a patient with X-linked ichthyosis associated with borderline mental retardation. J Dermatol Sci 45: 31-36, 2007.

3. Traupe $\mathrm{H}$ and Ropers $\mathrm{HH}$ : Cryptorchidism and hypogenitalism in X-linked recessive ichthyosis vulgaris. Hum Genet 60: 206, 1982.

4. Tiepolo L, Zuffardi O, Fraccaro M, di Natale D, Gargantini L, Müller CR and Ropers HH: Assignment by deletion mapping of the steroid sulfatase X-linked ichthyosis locus to Xp223. Hum Genet 54: 205-206, 1980.

5. Yen PH, Allen E, Marsh B, Mohandas T, Wang N, Taggart RT and Shapiro LJ: Cloning and expression of steroid sulfatase cDNA and the frequent occurrence of deletions in STS deficiency: Implications for X-Y interchange. Cell 49: 443-454, 1987.

6. Bonifas JM, Morley BJ, Oakey RE, Kan YW and Epstein EH Jr: Cloning of a cDNA for steroid sulfatase: Frequent occurrence of gene deletions in patients with recessive $\mathrm{X}$ chromosome-linked ichthyosis. Proc Natl Acad Sci USA 84: 9248-9251, 1987.

7. Crigler JF Jr and Najjar VA: Congenital familial nonhemolytic jaundice with kernicterus. Pediatrics 10: 169-180, 1952.

8. Petit FM, Gajdos V, Francoual J, Capel L, Parisot F, Poüs C and Labrune P: Allelic heterogeneity of Crigler-Najjar type I syndrome: A study of 24 cases. Clin Genet 66: 571-572, 2004.

9. Van der Veere CN, Sinaasappel M, McDonagh AF, Rosenthal P, Labrune $\mathrm{P}$, Odièvre $\mathrm{M}$, Fevery $\mathrm{J}$, Otte JB, McClean $\mathrm{P}$, Bürk G, et al: Current therapy for Crigler-Najjar syndrome type 1: Report of a world registry. Hepatology 24: 311-315, 1996.

10. Santos EM, Paula JF, Motta PM, Heinemann MB, Leite RC, Haddad JP, Del Puerto HL and Reis JK: Comparison of three methods of DNA extraction from peripheral blood mononuclear cells and lung fragments of equines. Genet Mol Res 9: 1591-1598, 2010.

11. Wilke K, Duman B and Horst J: Diagnosis of haploidy and triploidy based on measurement of gene copy number by real-time PCR. Hum Mutat 16: 431-436, 2000.

12. Li Y, Qu YJ, Zhong XM, Cao YY, Jin LM, Bai JL, Ma X, Jin YW, Wang H, Zhang YL and Song F: Two unrelated patients with rare Crigler-Najjar syndrome type I: Two novel mutations and a patient with loss of heterozygosity of UGT1A1 gene. J Zhejiang Univ Sci B 15: 474-481, 2014.

13. Murtaza G, Siddiq S, Khan S, Hussain S and Naeem M: Molecular study of X-linked ichthyosis: Report of a novel 2-bp insertion mutation in the STS and a very rare case of homozygous female patient. J Dermatol Sci 74: 165-167, 2014.

14. Fukami M, Kirsch S, Schiller S, Richter A, Benes V, Franco B, Muroya K, Rao E, Merker S, Niesler B, et al: A member of a gene family on Xp22.3, VCX-A, is deleted in patients with X-linked nonspecific mental retardation. Am J Hum Genet 67: 563-573, 2000.

15. Paige DG, Emilion GG, Bouloux PM and Harper JI: A clinical and genetic study of $\mathrm{X}$-linked recessive ichthyosis and contiguous gene defects. Br J Dermatol 131: 622-629, 1994. 
16. Van Steensel MA, Vreeburg M, Engelen J, Ghesquiere S, Stegmann AP, Herbergs J, van Lent J, Smeets B and Vles JH: Contiguous gene syndrome due to a maternally inherited $8.41 \mathrm{Mb}$ distal deletion of chromosome band Xp22.3 in a boy with short stature, ichthyosis, epilepsy, mental retardation, cerebral cortica heterotopias and Dandy-Walker malformation. Am J Med Genet A 146: 2944-2949, 2008.

17. Gohlke BC, Haug K, Fukami M, Friedl W, Noeker M, Rappold GA and Haverkamp F: Interstitial deletion in Xp22.3 is associated with $\mathrm{X}$ linked ichthyosis, mental retardation and epilepsy. J Med Genet 37: 600-602, 2000.

18. Lahn BT and Page DC: A human sex-chromosomal gene family expressed in male germ cells and encoding variably charged proteins. Hum Mol Genet 22: 311-319, 2000.

19. Canu G, Minucci A, Zuppi C and Capoluongo E: Gilbert and Crigler Najjar syndromes: An update of the UDP-glucuronosyltransferase 1A1 (UGT1A1) gene mutation database. Blood Cells Mol Dis 50: 273-280, 2013.
20. Zhang F, Gu W, Hurles ME and Lupski JR: Copy number variation in human health, disease and evolution. Annu Rev Genomics Hum Genet 10: 451-481, 2009.

21. Miller DT, Adam MP, Aradhya S, Biesecker LG, Brothman AR, Carter NP, Church DM, Crolla JA, Eichler EE, Epstein CJ, et al: Consensus statement: Chromosomal microarray is a first-tier clinical diagnostic test for individuals with developmental disabilities or congenital anomalies. Am J Hum Genet 14: 749-764, 2010.

22. Schaaf CP, Wiszniewska J and Beaudet AL: Copy number and SNP arrays in clinical diagnostics. Annu Rev Genomics Hum Genet 12: 25-51, 2011. 